\title{
Plasticity in the Olfactory System: Lessons for the Neurobiology of Memory
}

\author{
D. A. WILSON, A. R. BEST, and R. M. SULLIVAN \\ Department of Zoology \\ University of Oklahoma
}

\begin{abstract}
We are rapidly advancing toward an understanding of the molecular events underlying odor transduction, mechanisms of spatiotemporal central odor processing, and neural correlates of olfactory perception and cognition. A thread running through each of these broad components that define olfaction appears to be their dynamic nature. How odors are processed, at both the behavioral and neural level, is heavily dependent on past experience, current environmental context, and internal state. The neural plasticity that allows this dynamic processing is expressed nearly ubiquitously in the olfactory pathway, from olfactory receptor neurons to the higher-order cortex, and includes mechanisms ranging from changes in membrane excitability to changes in synaptic efficacy to neurogenesis and apoptosis. This review will describe recent findings regarding plasticity in the mammalian olfactory system that are believed to have general relevance for understanding the neurobiology of memory. NEUROSCIENTIST 10(6):513-524, 2004. DOI: $10.1177 / 1073858404267048$
\end{abstract}

KEY WORDS Olfaction, Plasticity, Memory, Learning, Perception

Odor perception is situational, contextual, and ecological. Odors are not stored in memory as unique entities. Rather, they are always interrelated with other sensory perceptions . . . that happen to coincide with them.

$$
\text { -Engen (1991, p 86-87) }
$$

The field of olfaction has experienced explosive growth over the past decade toward understanding the molecular events underlying transduction, mechanisms of spatiotemporal central processing, and neural correlates of olfactory perception and cognition. A thread running through each of these broad components that define olfaction appears to be their dynamic nature. How odors are processed, at both the behavioral and neural level, is heavily dependent on past experience, current environmental context, and internal state. The neural plasticity that allows this dynamic processing is expressed nearly ubiquitously in the olfactory pathway, from olfactory receptor neurons to the higher-order cortex, and includes mechanisms ranging from changes in membrane excitability to changes in synaptic efficacy to neurogenesis and apoptosis.

The olfactory system has proven to be an excellent model system for the study of the neurobiology of mem-

DAW and ARB were supported by grants from the National Institute on Deafness and Other Communicative Disorders and the Oklahoma Center for the Advancement of Science and Technology. RMS was supported by grants from the National Science Foundation and National Institute of Child Health and Human Development.

Address correspondence to: Donald A. Wilson, Department of Zoology, University of Oklahoma, Norman, OK 73019 (e-mail: dwilson@ou.edu). ory for several reasons. First, the olfactory system is phylogenetically highly conserved, and memory plays a critical role in many ecologically significant odorguided behaviors. Thus, many different animal models, ranging from Drosophila to primates, can be taken advantage of to address specific experimental questions. Second, the olfactory pathway is relatively short and perhaps simplified compared to mammalian thalamocortical systems, with second-order neurons projecting directly to a well-described trilaminar sensory cortex. Third, the olfactory system has very strong anatomical ties to the limbic system; for example, both the lateral nucleus of the amygdala and the hippocampal dentate gyrus are three synapses from olfactory receptor neurons in the nose. Finally, the olfactory system is heavily innervated by well-defined neuromodulatory systems known to be important for memory and neural plasticity.

This review will describe recent findings regarding plasticity in the mammalian olfactory system that we believe have general relevance for understanding the neurobiology of memory. Following a brief overview of olfactory system functional anatomy, we will review types of neural plasticity expressed in the olfactory system and then how these mechanisms relate to the diverse components of behavioral olfactory memory. Finally, we will attempt to identify some emergent principles of the neurobiology of olfactory memory and outline some potential future directions.

\section{Olfactory System Organization}

Very simply put, the primary olfactory pathway includes the olfactory receptor neurons in the nose (or antenna in many invertebrates), second-order neurons and affiliated 


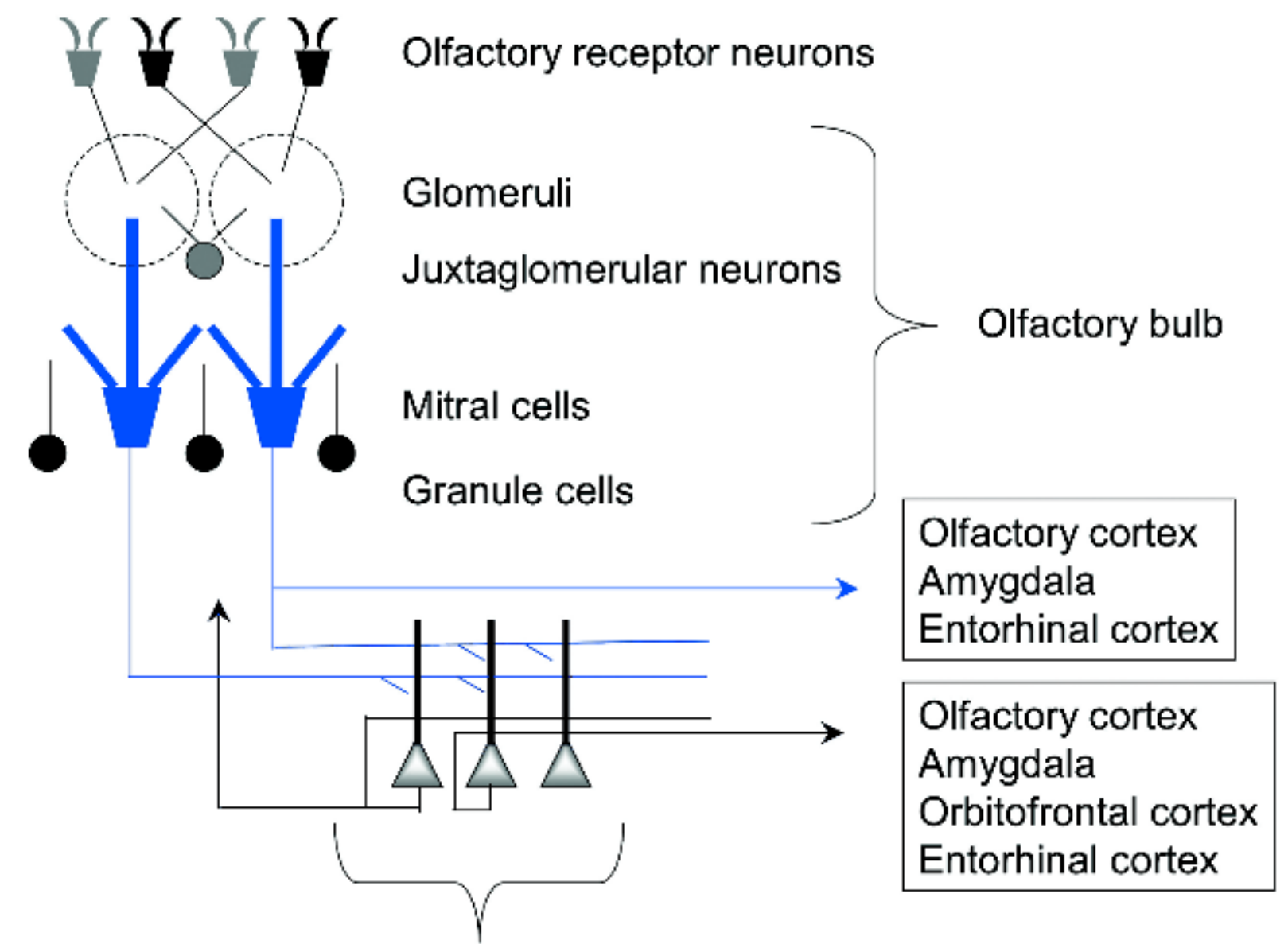

\section{Piriform cortex}

Fig. 1. Schematic representation of mammalian olfactory system circuitry. As described in the text, olfactory receptor neurons expressing one of many hundreds of different receptor proteins target single glomeruli in the olfactory bulb where they synapse on mitral cells. Mitral cells receive inhibitory inputs from juxtaglomerular neurons and granule cells and send their axons to the piriform cortex where they converge with mitral cells conveying input from different receptors. Higher-order connections include other regions of the olfactory cortex and limbic system structures.

circuitry within the olfactory bulb (or antennal lobe), and cortical neurons within the olfactory cortex (or mushroom bodies). However, in addition to this direct pathway, the mammalian olfactory system is strongly interconnected both with the higher-order cortex (e.g., orbitofrontal cortex) and with the limbic system including the amygdala, hippocampus, and perirhinal cortex. A number of recent, thorough reviews of olfactory system functional anatomy exist (Shipley and Ennis 1996; Haberly 2001; Mombaerts 2001; Savic 2002); thus, we will focus here on a few main points. First, although peripheral olfactory functional anatomy appears to emphasize analytical processing of odorants, ${ }^{1}$ central olfactory circuits very rapidly appear to shift toward architecture emphasizing synthetic processing of odorants. Second, there is extensive multimodal and/or modulatory input throughout the olfactory pathway that

1. The term odorant is used here to refer to an actual physicochemical stimulus, whereas the term odor is used to refer to a perceptual object, in accordance with Hudson (2000). appears to allow multisensory and state-dependent convergence with olfactory neurons. This convergent input further enhances synthetic processing of odorants with their context and their biological or learned significance (Fig. 1).

The olfactory receptor sheet of both vertebrates and invertebrates includes a population of receptor neurons that express scores (invertebrates) to hundreds (mammals) of different genes that encode olfactory receptor G-coupled proteins (Buck 1996). It appears that single olfactory neurons express a single receptor gene (Serizawa and others 2000) and that the ligands for these G-coupled receptors are generally submolecular components (or features) of an odorant, rather than the entire odorant molecule (Araneda and others 2000). Thus, transduction of a complex odor such as coffee will involve activation of many different olfactory receptor neurons expressing different olfactory receptor proteins, each binding submolecular components of the many different odorants in the headspace of the liquid in your cup. 
The process of putting the coffee back together begins with the remarkably precise projection of olfactory receptor neuron axons to the olfactory bulb. Despite the scattered mosaic of expression patterns for individual olfactory receptor genes in the olfactory receptor sheet, all receptor neurons expressing the same receptor gene converge onto about two small targets within the olfactory bulb called glomeruli. In the mammal, single mitral cells receive input from a single glomerulus. In addition to glutamatergic input from the receptor neurons, activity of mitral cells is also under the influence of juxtaglomerular interneurons, a large population of GABAergic interneurons called granule cells, as well as numerous centrifugal modulatory inputs such as norepinephrine and acetylcholine (ACh; Shipley and Ennis 1996). Granule cells not only appear to serve as feedback and lateral inhibitory interneurons but also are the target of the majority of centrifugal inputs to the bulb, including from the olfactory cortex and modulatory nuclei. With this circuitry, mitral cells appear to function largely as feature detectors, although the extensive interactions within and between glomeruli and via granule cells both enhances contrast between similar features (Yokoi and others 1995) and begins the process of feature interaction important for eventual synthesis.

Mitral cells are glutamatergic neurons that project to the olfactory cortex, cortical nucleus of the amygdala, and entorhinal cortex. The olfactory cortex is subdivided into several regions including the anterior olfactory nucleus, olfactory tubercle, and piriform cortex. Mitral cells terminate in the piriform cortex in patches of terminal arbors, synapsing on the distal apical dendrites of layer II and III pyramidal cells. Termination of mitral cells conveying information from olfactory receptors expressing one type of receptor gene overlaps with input from other receptor types, allowing for convergence of multiple receptors/odorant features onto single cortical neurons (Zou and others 2001). This convergence is dramatically enhanced by a dense network of intracortical association fibers, which terminate on the proximal apical dendrite and basal dendrites of layer II/III pyramidal cells. The cortical afferent and association fiber systems, in addition to being anatomically segregated on the cortical neuron dendrite, appear also to have significant differences in physiology, including plasticity and modulatory control, as described below. This highly associative network is hypothesized to be critical for the final synthesis of the multiple odorant features extracted and refined peripherally into unique odor perceptual objects (Haberly 2001). In addition, given the variety of nonolfactory and modulatory inputs to the piriform cortex, the cortical associative network is hypothesized to play a role beyond odor processing per se and includes contextual, hedonic, and memorial components of the synthetic odor perception (Haberly 2001).

The multimodal, contextual nature of cortical odor processing is also expressed in the orbitofrontal cortex (Schoenbaum and Eichenbaum 1995; Rolls 2000). The orbitofrontal cortex receives some direct input from piriform layer II/III cortical neurons, as well as thalamic
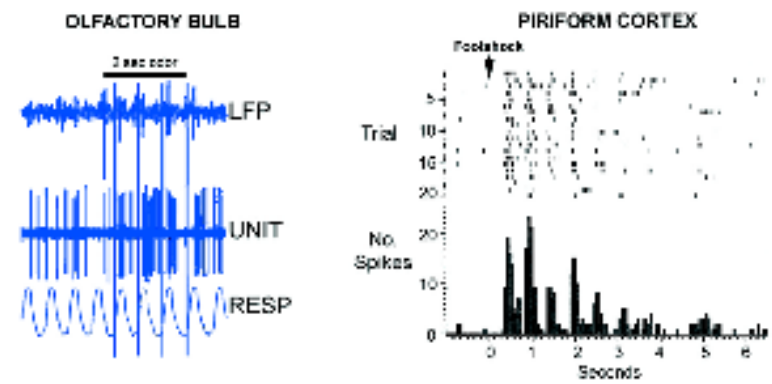

Fig. 2. Examples of single-unit responses to odor and to nonodor stimulation in the rat olfactory system. Left, Local field potential (LFP; filtered for $\beta$ wave activity, 15-45 Hz) and mitral cell single-unit activity in response to a 2-sec odor pulse. Respiration as monitored by chest wall movements is the bottom trace. Note the increase in both firing rate and temporal correlation with the respiratory cycle during the odor stimulus. The firing bursts correspond to $\beta$ frequency bursts in the LFP. Similar responses to odor can be observed in the piriform cortex (not shown). Right, Piriform cortex single-unit response to repeated 200-ms footshock in a urethane anesthetized rat. Rasterplot and cumulative peristimulus histogram show footshock-evoked increase in cortical firing rate, but note that firing is selectively increased in phase with respiration to produce bursts on each inhalation (respiration not shown). This suggests that rather than directly responding to the footshock, the footshock enhances responsiveness to olfactory input (Bouret, Wilson, and Sara, unpublished observations).

input from the medial dorsal thalamic nucleus. Neurons in the orbitofrontal cortex have been shown to respond to specific odors (Critchley and Rolls 1996), specific odortaste compounds (de Araujo and others 2003), and odorcontext cues (Ramus and Eichenbaum 2000). It should be noted that the synthetic output of both piriform and orbitofrontal cortical circuits is fed back to the olfactory bulb, perhaps with a role similar to corticothalamic feedback in other sensory systems (Fig. 2).

Neurons in both the olfactory bulb and piriform cortex also project directly to the amygdala and entorhinal cortex, the latter of which serves as a sensory gateway into the hippocampal formation. The role of the hippocampal formation and the amygdala in memory for olfactory cues has been well described (Staubli and others 1995; Eichenbaum 1998; Rosenkranz and Grace 2002) and will not be a focus of this review.

As noted above, the olfactory pathway is heavily innervated by modulatory systems known to regulate plasticity and memory in the olfactory system as elsewhere. Both the olfactory bulb and cortex receive a strong cholinergic input from the horizontal limb of the diagonal band of Broca (HLDB), which itself is responsive to olfactory input (Linster and Hasselmo 2000). This creates a potentially interesting feedback loop in which cholinergic modulation of olfactory processing is itself partially under olfactory control. Norepinephrine from the nucleus locus coeruleus also heavily innervates the olfactory bulb and cortex (Shipley and Ennis 1996) and, as described below, plays a critical function in several forms of olfactory system plasticity and memory. 


\section{Modulation of sensory afferent input}

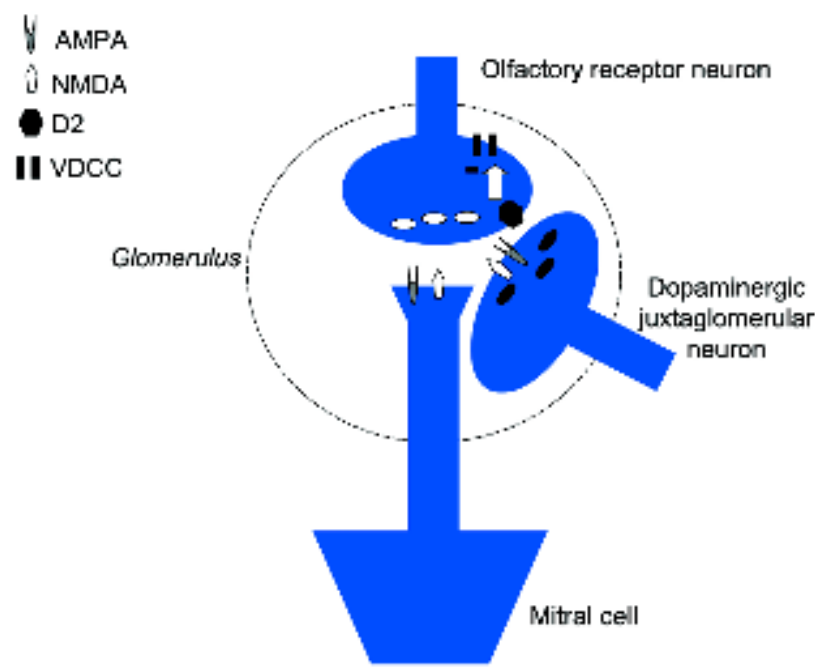

Modulation of cortical afferent input

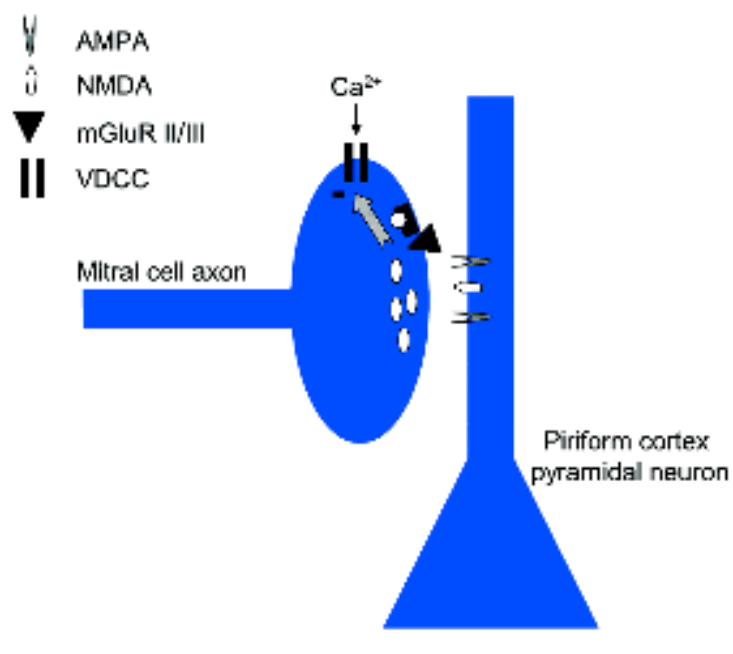

Fig. 3. Examples of two mechanisms of short-term synaptic plasticity in the olfactory system. Left, Afferent input to the olfactory bulb can be regulated by dopaminergic feedback to D2 receptors on the olfactory receptor axons. As described in the text, dopamine levels are modulated by odor stimulation such that reduced odor stimulation reduces dopamine expression and releases olfactory nerve axon terminals from presynaptic inhibition. This could produce an activity-dependent regulation of afferent input efficacy. In the piriform cortex (right), mitral cell axon transmitter release is modulated by mGluR autoreceptors. During periods of intense input (e.g., prolonged odor stimulation), transmitter release is reduced, potentially producing cortical odor adaptation.

\section{Olfactory System Plasticity}

The olfactory system has at its disposal an extremely wide range of tools for storing information. In response to learning and odor experience, olfactory circuits display short- and long-term synaptic plasticity, nonsynaptic plasticity of membrane biophysics, morphological changes in dendritic complexity, and experiencedependent neurogenesis and apoptosis. This section reviews the forms of plasticity that have been described in the olfactory system and the conditions involved in inducing that plasticity. The next section then describes different forms of behavioral olfactory memory and what is known about their relationship to the neural plasticity toolkit described here.

Synaptic plasticity has been hypothesized to be a mechanism of storing information in the nervous system as long as synapses have been known to exist. Chemical synaptic transmission is dynamic, reflecting both recent and, at some synapses, more historical activity patterns. Electrical synapses can also be modulated by experience. In the olfactory system, short-term synaptic plasticity occurs in both the olfactory bulb and piriform cortex to shape current activity in light of recent patterns of input. This short-term synaptic plasticity may be important in adaptation to repeated or prolonged stimulation and in fine-tuning sensory processing to deal with patchy, unpredictable odor plumes.

Anterior piriform cortical neurons rapidly adapt to novel odors, despite relatively maintained input from their excitatory afferent, glutamatergic mitral cells (Wilson 1998a). A similar, rapid decrease in odorevoked spiking occurs in the insect mushroom body neu- rons (Stopfer and Laurent 1999). Recent work has demonstrated that in the rat, this cortical adaptation may be primarily due to activation of presynaptic metabotropic glutamate receptors (mGluR) on mitral cell axons (Best and Wilson 2004). This synaptic depression has a duration of less than 2 min following a 50-sec odor exposure, as does the reduction of cortical responses to subsequent stimulation with that odor (Wilson 1998b). Blockade of mGluR II/III receptors prevents afferent synaptic depression and cortical adaptation to odors. This mechanism of cortical adaptation allows maintained input to other, nonexperienced afferent inputs (the depression is homosynaptic). In addition, it appears to allow a mechanism of dishabituation in cases in which contingencies or context change and it becomes adaptive to begin responding to the stimulus again. The molecular cascade regulated by $\mathrm{mGluR}$ II/III receptors interacts with that activated by noradrenergic $\beta$ receptors (Cai and others 2001), such that $\beta$-receptor activation can reverse the effects of $\mathrm{mGluR}$ activation. In accordance, the synaptic depression believed important for cortical adaptation can be prevented by activation of noradrenergic $\beta$ receptors (Best and Wilson 2004; Fig. 3).

Short-term changes in synaptic strength may also be involved in dynamic coding of odors by olfactory bulb circuits. Both temporal (Meredith 1992; Kauer 1988; Perez-Orive and others 2002) and spatial (Spors and Grinvald 2002) patterns of olfactory bulb circuit responses to odors change over the course of even very brief stimulus presentations, suggesting a dynamic remodeling of local circuits, potentially involving changes in synaptic efficacy. For example, in mammals, 
olfactory receptor axon terminals express dopamine D2 receptors, and a local population of juxtaglomerular neurons express dopamine (Shipley and Ennis 1996). Activation of D2 receptors in the olfactory bulb reduces transmitter release from olfactory receptor axons, effectively reducing the gain of afferent input (Wachowiak and Cohen 1999; Ennis and others 2001). Conversely, a reduction in olfactory stimulation (odor deprivation) causes an $N$-methyl-D-aspartate (NMDA) receptordependent (Puche and Shipley 1999) decrease in dopamine expression in these neurons (Cho and others 1996), which effectively enhances the responsiveness of mitral cells to subsequent stimulation (Wilson and Sullivan 1995). Thus, as with piriform cortical afferents, recent levels of odor stimulation can be remembered and adjusted for through a presynaptic regulation of transmitter release. This mechanism may function over a wide time scale, monitoring activity levels ranging from seconds (Ennis and others 2001) to weeks (Cho and others 1996).

Long-term synaptic plasticity is also evident in the olfactory system. In vertebrates, olfactory receptor neurons, mitral cells, and piriform cortical pyramidal neurons are all glutamatergic, and most postsynaptic targets of these neurons express both NMDA and non-NMDA receptors (Shipley and Ennis 1996). Recent in vitro work suggests that olfactory receptor neuron-to-mitral cell synapses can express long-term potentiation (LTP) following high-frequency activation (Ennis and others 1998). The precise role of LTP at this synapse in odor coding or memory is unclear; however, it could shift the balance of excitation and inhibition within a glomerulus to enhance mitral cell output from previously activated glomeruli. Past experience can shift mitral cell odor feature tuning toward the familiar odorants (Fletcher and Wilson 2003), which could contribute to perceptual learning and enhanced odor acuity.

The mitral cell-granule cell synapse has also been strongly implicated as supporting long-term plasticity. Granule cells express both NMDA and non-NMDA receptors and express high levels of CAMKII (Zou and others 2002) characteristics that are common in neurons capable of experience-dependent plasticity like LTP. Following associative conditioning, there is a change in the ratio of glutamate release (presumably from mitral cells) to GABA release (presumably from granule cells) in favor of heightened GABA release (Brennan and others 1998). This has been interpreted as an increase in mitral cell to granule cell synaptic efficacy induced by associative conditioning. In young rats, associative odor conditioning enhances mitral cell-suppressive responses to the learned odor (Wilson and others 1985), which has also been hypothesized to result from enhanced mitral to granule cell synaptic transmission.

Experience-induced changes in the balance of synaptic excitation and inhibition within the olfactory bulb circuit not only can influence the probability or rate of mitral cell action potential output but also can influence spike timing and firing synchrony between ensembles of mitral cells. This is evident from pharmacological manipulations in invertebrates (MacLeod and Laurent 1996) as well as in work from transgenic mice (Nusser and others 2001). Thus, an enhancement in granule cell GABAergic feedback to mitral cells in transgenic mice (which lack GABAergic inhibition of granule cells) enhances circuit synchrony as evidenced by increased oscillatory power in the olfactory bulb local field potential (LFP; Nusser and others 2001). Increased synchrony of olfactory bulb output neurons could further enhance the probability of LTP-like changes in synaptic strength within the olfactory bulb and/or within olfactory bulb efferent structures such as the piriform cortex. In accord with this interpretation, changes in olfactory bulb LFP oscillatory power in the $\beta(15-35 \mathrm{~Hz})$ and $\gamma(35-90 \mathrm{~Hz})$ frequency ranges are strongly correlated with odor learning and response to familiar odors (Grajski and Freeman 1989; Ravel and others 2003; Martin and others 2004; see below).

During odorant sniffing, mitral cells often fire in bursts of activity, with instantaneous firing frequencies of more than $100 \mathrm{~Hz}$, and in individual bursts in phase with the respiratory cycle, which occurs at a rate of 2 to $12 \mathrm{~Hz}$. These firing parameters are nearly identical to the ideal parameters for inducing LTP in limbic circuits (Jung and others 1990). LTP of mitral cell afferent synapses in piriform cortex, however, appears less robust than LTP of intracortical association fiber synapses (Kanter and Haberly 1990; Saar and others 1999), although when afferent input is activated in association with intracortical fibers and GABA blockade (Kanter and Haberly 1993) or in the context of an olfactory learning paradigm (Roman and others 1993), LTP of afferent synapses can be induced. LTP of both afferent and association fiber synapses is NMDA receptor dependent (Kanter and Haberly 1990). The cortical feedback pathway to the olfactory bulb also displays experience-dependent plasticity (Patneau and Stripling 1992), which may play a very important role in shaping subsequent response patterns to familiar odors.

Both resting strength and LTP-like plasticity of association synapses (but less so of afferent synapses) can be modulated by nonolfactory inputs such as ACh from the HLDB (Linster and Hasselmo 2001) and norepinephrine from the locus coeruleus (Linster and Hasselmo 2001). For example, ACh muscarinic receptor agonists enhance association synapse LTP. This modulation of plasticity could play an important role in attentional or learninginduced changes in cortical processing and information storage, as discussed below.

As discussed above, changes in synaptic strength, particularly in association synapse strength, may affect not only cortical neuron firing probability or rate but also temporal patterning and synchrony of cortical ensembles. Conditioning or experience-dependent changes in LFP oscillatory power in the $\beta$ and $\gamma$ frequency bands in piriform cortical circuits have been demonstrated (Bressler 1988).

In addition to changes in synaptic strength, several forms of nonsynaptic plasticity have been described in the olfactory system. These include changes in neural 
membrane biophysics, changes in dendritic morphology, and experience-dependent cell survival. For example, Barkai and colleagues have found an increase in excitability of piriform cortex pyramidal cells after odor discrimination learning (Saar and Barkai 2003). This increase in excitability appears to be due to a decrease in both neuronal adaptation and in a $\mathrm{Ca}^{2+}$-dependent afterhyperpolarization (Saar and others 2001). Interestingly, these learning-induced changes in intrinsic properties of piriform cortical pyramidal cells mimic and interact with the effects of the LTP enhancer ACh (Saar and Barkai 2003), suggesting that once the circuit has begun to learn odor discriminations, subsequent discrimination learning may be facilitated. This "learning to learn" is referred to as set or rule learning and is very robust in rat olfaction (Slotnick and others 2000).

Odor experience can also modify the morphology and survival of central olfactory neurons. As has been described in many other sensory systems, dendritic morphology of principal neurons in both the olfactory bulb (Brunjes 1994) and piriform cortex (Wilson and others 2000) can be regulated by the level of odor exposure. The precise role of dendritic morphological changes in odor memory has not been directly examined, although it could allow changes in connectivity of neurons within an ensemble.

Neurogenesis of olfactory bulb granule cells occurs throughout life (Rosselli-Austin and Altman 1979), and survival has recently been shown to be dependent on odor experience, with odor deprivation reducing survival (Frazier-Cierpial and Brunjes 1989; Najbauer and Leon 1995) and odor learning or exposure enhancing survival (Rochefort and others 2002). Given the importance of granule cells in odor coding and plasticity described above, it has been hypothesized that odor experience could selectively promote survival of newly generated granule cells into odorant-selective circuits, enhancing memory and discriminability for those odorants (Lledo and Gheusi 2003). Odor exposure during early development may also regulate survival of juxtaglomerular neurons near odor-specific glomeruli (Woo and Leon 1991), although this appears to occur only during early development. Finally, odor exposure is also necessary for survival of a select class of piriform cortical neurons (Leung and Wilson 2003), although the precise function of these neurons is unknown.

\section{Neurobiology of Olfactory Memory}

Olfactory memory, as with memory in general, can be divided into several subclasses (Clark and others 2002). These include 1) implicit memory such as habituation, sensitization, classical conditioning, and perceptual learning; 2) explicit memory such as delayed-match-tosample paradigms, spatial memory, and paired-associate memory; and 3) set learning or learning to learn. Although specific behavioral paradigms are used to test each of these forms of memory and evidence exists for specific neural mechanisms underlying them, it must be emphasized that in a given context or situation, several of these forms of memory may be evoked at the same time. It should also be noted that although this section will focus on mechanisms of memory within the olfactory system, the types of experiences involved may produce changes in a diverse collection of brain circuits (cortical and subcortical) that may ultimately be required for expression of the acquired memory.

\section{Implicit Memory}

Implicit memory includes habituation, sensitization, classical conditioning, and perceptual learning (Clark and others 2002). Habituation is a decrease in responsiveness to repeated or prolonged stimulation, relatively specific to the repeated stimulus, and subject to dishabituation. Habituation and adaptation allow sensory systems to filter background or currently nonsignificant stimuli while maintaining responsiveness to novel stimuli. Habituation to odors could involve olfactory receptor adaptation and/or central mechanisms. In many thalamocortical sensory systems, cortical neurons adapt more rapidly and completely than more peripheral neurons. This could allow for rapid dishabituation if the contingencies change and the stimulus becomes potentially important. Similarly, although in the olfactory system both receptor neurons (Chaput 2000; Zufall and Leinders-Zufall 2000) and mitral cells adapt to odors (Potter and Chorover 1976; Scott 1977; Wilson 2000), piriform cortical neurons adapt much more rapidly and completely following either prolonged or repeated odor stimulation (Wilson 2000). Cortical adaptation is also more selective (displaying less cross-adaptation) to familiar odors than is mitral cell adaptation (Wilson 2000).

The mechanism of short-term depression of cortical afferent synapses described above may contribute to behavioral habituation. Thus, during prolonged odor exposure (e.g., a background room odor), olfactory receptor neurons begin to slowly adapt through a defined $\mathrm{Ca}^{2+}$-dependent mechanism (Zufall and others 1991), mitral cells begin to slowly adapt through an unknown mechanism, and, at the same time, piriform cortical neurons rapidly adapt, in part through a mGluRII/IIImediated decrease in glutamate release from mitral cell axons (Best and Wilson 2004). However, if the odor becomes significant or arousal level increases (dishabituation), central olfactory system responding may return (Scott 1977). One mediator of this dishabituation could be norepinephrine from the locus coeruleus. Norepinephrine has at least two actions related to adaptation in the olfactory system. First, norepinephrine can increase mitral cell responsiveness to olfactory nerve input (Jiang and others 1996). Thus, in rats, a tail pinch can reinstate mitral cell responses to an adapted stimulus (Scott 1977), perhaps in part via a noradrenergic enhancement of mitral cell responsiveness to olfactory nerve input. In concert with this change in the olfactory bulb, norepinephrine can modify/enhance piriform cortical neuron responses to odors (Bouret and Sara 2002; Best and Wilson 2004). In fact, norepinephrine can 
block afferent synaptic depression to cortical neurons, perhaps via a direct interaction between noradrenergic $\beta$ receptor activation and mGluRII/III receptors (Cai and others 2001). ACh has also been implicated as modulating odor habituation (Hunter and Murray 1989), although precise mechanisms have not been determined.

As mentioned above, the extent of cross-adaptation between similar odors is dependent on whether those odors are familiar. In fact, as in other sensory systems, prior experience can enhance olfactory acuity, enabling greater discrimination of similar stimuli (Fletcher and Wilson 2002; Linster and others 2002), a process called perceptual learning (Wilson and Stevenson 2003). Several of the mechanisms discussed above may contribute to olfactory perceptual learning. For example, simple odor exposure modifies olfactory bulb circuit and mitral cell single-unit responses to subsequent odor presentation at both a very rapid (msec) time scale (Kay and Laurent 1999; Spors and Grinvald 2002) and a long (hours to days) time scale (Buonviso and others 1998; Fletcher and Wilson 2003). The rapid modification can occur within a single brief odor presentation and may reflect a dynamic fine-tuning of odor-responsive ensembles (Kay and Laurent 1999; Spors and Grinvald 2002). However, a long-term cascade is also generated that ultimately produces more permanent changes in subsequent mitral cell odor responses. Of most relevance to perceptual learning, mitral cell odor-receptive fields can shift toward the familiar odor, at least over short distances and along odorant feature dimensions such as carbon chain length (Fletcher and Wilson 2003). These experienceinduced changes in mitral cell odorant-receptive fields have been hypothesized to be due to mechanisms related to either changes in olfactory receptor synaptic efficacy (i.e., LTP), changes in mitral cell-inhibitory interneuron synaptic efficacy, and/or changes in efficacy in cortical feedback. In support of changes in inhibitory connectivity, simple odor exposure in invertebrates results in a progressive increase in LFP oscillatory power (Stopfer and Laurent 1999), a measure noted above as being sensitive to local interneuron synaptic inhibition. Regardless of the mechanism of receptive field change, the results suggest that familiar odorant features are encoded differently than are novel features, with perhaps an enhanced representation of familiar features.

Simple odor exposure (odor enrichment) also enhances survival of newborn granule cells in the adult mouse olfactory bulb (Rochefort and others 2002). Animals with enrichment-induced increases in granule cell number also have enhanced odor memory (Rochefort and others 2002). This again points to the potentially critical role of these inhibitory interneurons in odor memory, as discussed below.

In addition to changes in the olfactory bulb, perceptual learning is also associated with changes within the piriform cortex. For example, piriform cortical neurons show similar levels of cross-adaptation to novel odors as mitral cells. However, after at least $50 \mathrm{sec}$ of familiarization to a previously novel odor, the ability of cortical neurons to discriminate between molecularly similar odorants is greatly enhanced (Wilson 2003). Both behavioral perceptual learning and this change in cortical discrimination can be blocked by the cholinergic muscarinic receptor antagonist scopolamine (Fletcher and Wilson 2002; Wilson 2001). Muscarinic receptor activation enhances LTP of association fiber synapses (Hasselmo and Barkai 1995); thus, scopolamine should reduce cortical synaptic plasticity. Based on theoretical and computational modeling work, it has been hypothesized that activity-dependent enhancement of intracortical association fiber synapses allows patterns of odorant features to be synthesized by cortical ensembles, creating perceptual odor objects from the collection of odorant features extracted by the periphery (Haberly 2001). Once these odor objects have been synthesized, discriminating objects from each other (rather than collections of overlapping features) is enhanced, accounting for enhanced acuity for familiar odors. It should be noted that $\mathrm{ACh}$ has also been implicated in the effects of experience on odor coding in the olfactory bulb (Linster and Cleland 2002) to enhance distinctiveness of evoked feature patterns.

Similar changes in both the olfactory bulb and piriform cortex have been observed in association with olfactory classical conditioning, although in this model, norepinephrine has been most thoroughly examined as playing a permissive or modulatory role in circuit plasticity. Classical associative conditioning, in which an odorant signals the occurrence of, or is temporally paired with, an unconditioned or biologically significant stimulus, has been examined in several paradigms. These include the associative conditioning underlying neonate recognition of maternal odors in rats, maternal recognition of neonate odors in sheep, learned recognition of mates in mice, and simple aversive conditioning. The results of all of these paradigms implicate similar associated neural mechanisms. First, the olfactory bulb (or accessory olfactory bulb) is modified by the conditioning, generally with an enhancement of synaptic inhibition relative to excitation. Thus, for example, there is an enhancement of the ratio of GABA release to glutamate release in the accessory olfactory bulbs of recently mated mice in response to the learned odor (Brennan and others 1995; Kaba and Nakanishi 1995). Furthermore, there is an increase in suppressive responses to the learned odor in mitral cells near the odorspecific activated glomeruli and a relative decrease in excitatory responses (Wilson and Leon 1988). Finally, there is enhancement (or at least modification) of LFP oscillations that are known to be influenced by synaptic inhibition (Grajski and Freeman 1989; Ravel and others 2003).

Importantly, all three of these circuit changes, as well as the learned behaviors, are dependent on norepinephrine release in the olfactory bulb paired with the conditioned odor during learning (Sullivan and others 1989; Sullivan and Wilson 1994). For example, infusion of norepinephrine or receptor agonists directly into the olfactory bulb is sufficient to induce changes in olfactory bulb odor-evoked responses and behaviorally 


\section{Classical conditioning in olfactory bulb}

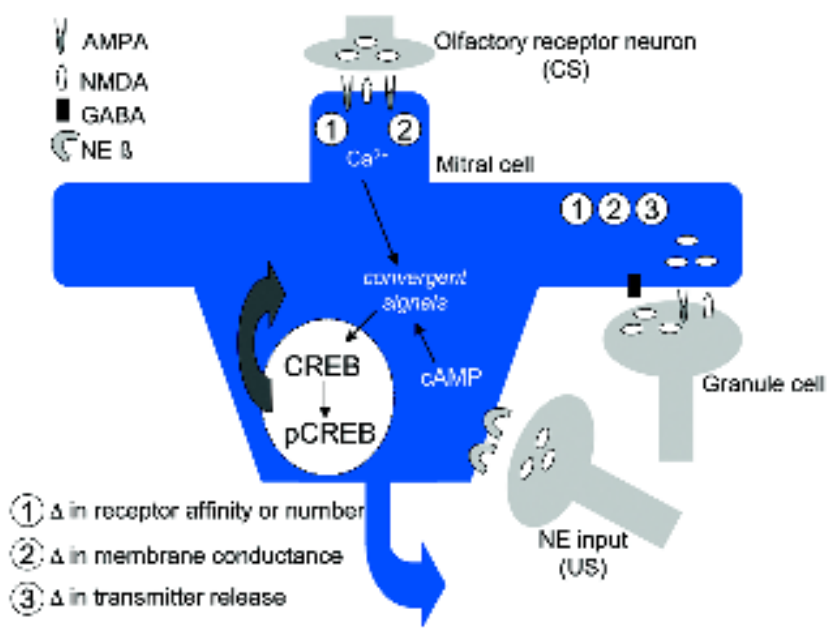

Rule learning in piriform cortex

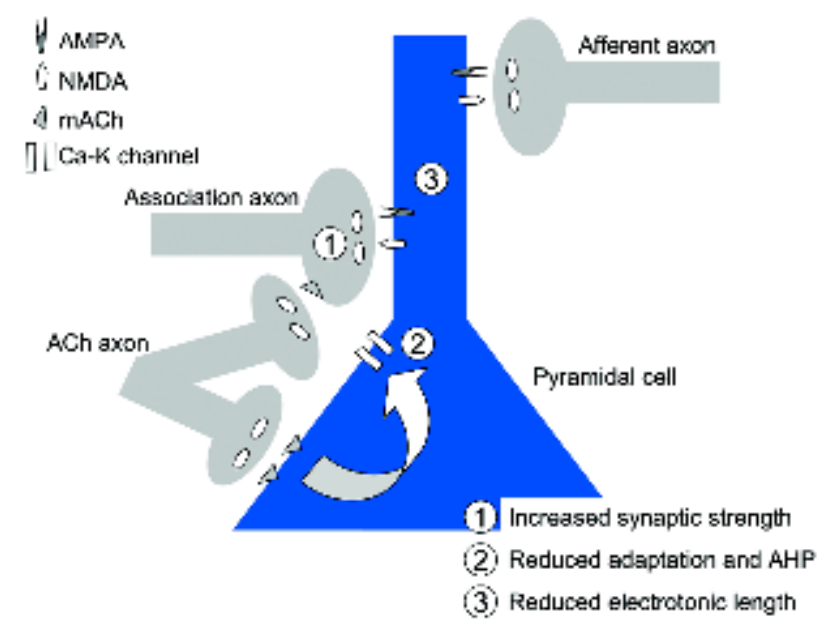

Fig. 4. Examples of two mechanisms of long-term plasticity in the olfactory system. Left, In the olfactory bulb, mitral cells receive convergent odor and centrifugal inputs such as norepinephrine from the locus coeruleus. When an odor is paired with an arousing (locus coeruleus activating) unconditioned stimulus, the combination can induce an intracellular second messenger cascade activating CREB and modifying gene transcription for long-term changes in cell function. Right, In the piriform cortex, association of odor-evoked association fiber activity with cholinergic input can produce a variety of synaptic and membrane biophysical changes that result in modified pyramidal cell responses to subsequent odor stimulation and heightened probability of further plasticity (see text).

expressed memories of those odors (Gray and others 1986; Sullivan and others 2000). These results suggest that norepinephrine may convey information about the unconditioned stimulus to the olfactory bulb for convergence with odor-specific input. Both granule cells and mitral cells express noradrenergic receptors, and norepinephrine modulates the mitral-granule cell reciprocal synapse (Trombley and Shepherd 1992); however, recent work suggests a critical site of convergence between norepinephrine and odor input may be mitral cells. As shown in Figure 4, activation of mitral cell $\beta$ noradrenergic receptors, combined with glutamatergic odor input, elevates cAMP levels that ultimately result in phosphorylation of CREB and subsequent protein synthesis changes that modify mitral cell function (Yuan and others 2000; Yuan, Harley, and McLean 2003). Hypothesized changes include mitral cell sensitivity to input and synaptic output that could account for the observed changes in olfactory bulb circuit function described above. Importantly, experimental modulation of cAMP levels (Yuan, Harley, and McLean 2003) or CREB phosphorylation (Yuan, Harley, Darby-King, and others 2003) produce the expected changes in learned behavior. ${ }^{2}$ Additional work will be needed to determine the effect of these manipulations on olfactory circuit function, but these results emphasize the critical role of centrifugal input to the olfactory bulb (and olfactory second-order neurons) in shaping olfactory processing and memory.

2. Importantly, a similar molecular cascade has been identified in mushroom body neurons of Drosophila during odor conditioning (Connolly and others 1996).
The effects of simple associative conditioning on the piriform cortex have been less well studied (Roman and others 1987; Litaudon and others 1997; Datiche and others 2001; Zinyuk and others 2001; Saar and others 2002). Depending on the specific behavioral paradigm used, learning a simple odor discrimination can enhance both afferent synaptic strength (Roman and others 1987) and association fiber synaptic strength (Saar and others 2002), although the association fiber changes may be longer lasting and may be most robust in the posterior piriform cortex compared to the anterior piriform cortex (Litaudon and others 1997). In addition to changes in synaptic strength, membrane properties are modified in layer II piriform cortical neurons, resulting in enhanced excitability as described above (Saar and others 2002). However, these changes in membrane excitability may be more important in priming the cortex for learning subsequent odors than in storing information about specific stimuli (see below; Saar and Barkai 2003). Both the neural plasticity and the behavioral expression induced by training can be modulated with ACh (De Rosa and Hasselmo 2000; Saar and others 2001).

In an associative conditioning context, the observed changes in association fiber synaptic strength have been hypothesized to involve not only odor-specific information but also contextual and perhaps multimodal information (Haberly 2001; Linster and Hasselmo 2001). Thus, the "object" representation of odors built up through cortical associative plasticity may include factors well beyond the simple physicochemical features of the odorant itself (Schoenbaum and Eichenbaum 1995; Fig. 5). 


\section{Perceptual Learning}

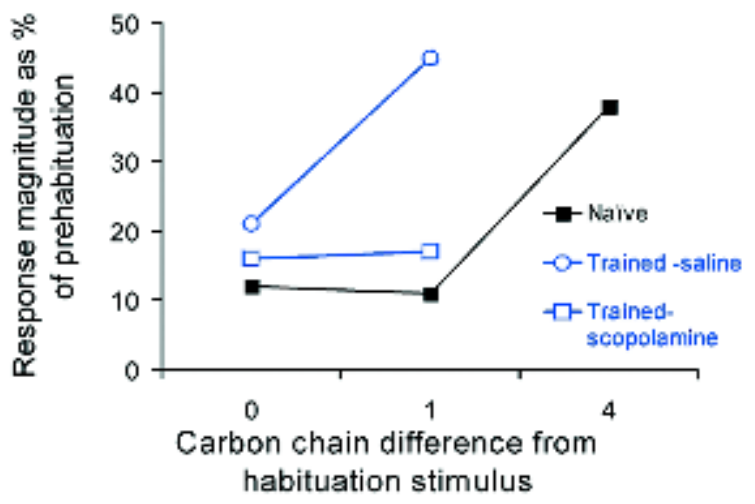

Rule Learning

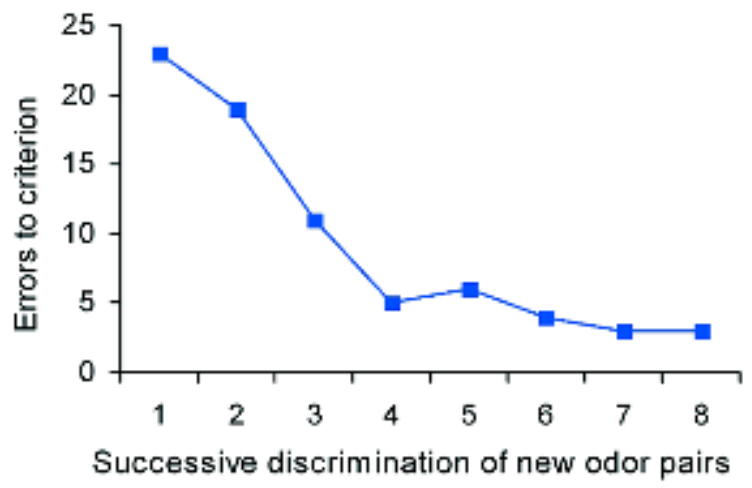

Fig. 5. Examples of olfactory rule/set learning and olfactory perceptual learning behavioral data. Top, After becoming familiar with an odor, that odor becomes more distinct from molecularly similar odors (perceptual learning). Naive rats are unable to discriminate unfamiliar ethyl esters varying by a single carbon, whereas after a familiarization procedure, they are capable of making this discrimination. Blockade of ACh muscarinic receptors with scopolamine during the familiarization training prevents perceptual learning. Bottom, As rats are given repeated discrimination training with new odor pairs (e.g., discriminate apple from banana, then discriminate coffee from mint, etc.), they significantly improve their learning speed, such that often only single trials are required for subsequent errorless performance after a few odor pairs. Data adapted from Slotnick and others (2000).

\section{Explicit Memory}

Explicit memory includes memory for facts that can be cognitively manipulated and is evidenced in behavioral paradigms such as delayed-match-to-(non)sample and paired-associated memory. Much less is known about specific neural mechanisms of these types of olfactory memory, although brain regions involved have been identified. Neurons in the piriform cortex, orbitofrontal cortex, perirhinal cortex, ventral striatum, and hippocampus may contribute to memory and performance in explicit memory tasks (Slotnick 2001). Work with these paradigms emphasizes that 1) explicit memory is distributed across both primary sensory and higher-order regions (e.g., Schoenbaum and Eichenbaum 1995); 2) even neurons in traditionally primary sensory areas, such as piriform cortex, may respond to multiple, nonolfactory aspects of the conditioning paradigm such as stimulus hedonics and nonodor task-related stimuli (although see the caveat in Fig. 2; Alvarez and Eichenbaum 2002; Critchley and Rolls 1996; Lipton and others 1999); and 3) although not specifically examined in this work, it is likely that much of the implicit memory-associated changes in the olfactory system outlined above also occur in these paradigms.

\section{Rule Learning}

Finally, rule learning or set learning is an increase in learning performance with each new stimulus discrimination to be made. Thus, for example, if a rat takes 50 trials to learn to discriminate odor A from odor B, subsequently the same rat may take only 30 trials to learn odor $\mathrm{C}$ versus odor $\mathrm{D}$ and eventually attain one trial learning on later odor pairs (Slotnick and others 2000). Recent work in the piriform cortex has provided a potential mechanism for rule learning. Barkai and colleagues have found that for several days after learning an odor discrimination task, piriform cortical neurons demonstrate increased excitability, decreased afterhyperpolarizations, and a potential electrotonic shortening of apical dendrites (Saar and Barkai 2003). This combination of biophysical changes results in cortical cells that may fire longer and more intensely to sensory input, making associative synaptic plasticity more likely and thus facilitating learning of subsequent odor discriminations. A similar combination of effects can be induced in naive cortical cells with ACh muscarinic receptor activation (Saar and others 2001), and in fact, once these changes are induced through learning, ACh becomes less effective (Saar and others 2001). Similarly, ACh modulation of odor discrimination learning weakens as rule learning progresses (Saar and others 2001). These data suggest that initial learning primes the cortex into a "sensitive state" for heightened learning of new discriminations.

\section{Emergent Principles and Future Directions}

The work described here suggests that both associative and nonassociative implicit memory are correlated with changes in both olfactory bulb and piriform cortical circuits. Thus, the neural substrates or consequences of even very simple memory are distributed in multiple brain circuits. Just as there are multiple circuits involved in odor memory, so are there multiple synaptic and nonsynaptic mechanisms for neural change. These changes result not only in modified behavioral responses to the learned odor but also in modified discriminability and perception of the learned odor. Learned changes occur as early as second-order neurons of the sensory pathway, and inhibitory interneurons play a critical role in expression of learned changes in circuit function. Finally, mod- 
ulatory inputs, such as norepinephrine and $\mathrm{ACh}$, are critical for modulating neural plasticity and behavioral change. Explicit and set learning are also associated with changes in the piriform cortex and, again, are under the direct influence of neuromodulators. In all three types of memory (implicit, explicit, and set learning), the specific synaptic and neural changes involved not only modify probability or rate of single-unit firing but also affect circuit function as a whole by changing the dynamic between ensembles of neurons - to date, most thoroughly examined in the olfactory system through LFP oscillations.

The results described here (and many others we were unable to include) suggest several avenues for future work, three of which will be briefly highlighted. First, as in other sensory systems, the olfactory system has extensive feedback pathways and is the recipient of multiple modulatory and descending inputs. Understanding how these pathways contribute to sensory coding and memory will be critical to understanding this system. Second, as shown so well in the olfactory bulb where there is an output neuron-to-interneuron ratio of approximately 1:50, interneurons are critical in experienceinduced change and memory, well beyond notions of lateral inhibition. Future work on how these neurons shape olfactory circuit function could be an excellent way to understand memory functions of interneurons in other systems. Finally, although important work remains in understanding how individual neurons are shaped by experience, more focus must be placed on understating how individual neurons function within ensembles and how those ensembles are affected by learning. The relative simplicity of the olfactory system, the important role of plasticity and experience-induced change in olfactory function, and the power of multiple, highly conserved model systems have made olfaction an ideal system for the study of the neurobiology of memory.

\section{References}

Alvarez P, Eichenbaum H. 2002. Representations of odors in the rat orbitofrontal cortex change during and after learning. Behav Neurosci 116:421-33.

Araneda RC, Kini AD, Firestein S. 2000. The molecular receptive range of an odorant receptor. Nat Neurosci 3:1248-55.

Best AR, Wilson DA. 2004. Coordinate synaptic mechanisms contributing to olfactory cortical adaptation. J Neurosci 24:652-60.

Bouret S, Sara SJ. 2002. Locus coeruleus activation modulates firing rate and temporal organization of odour-induced single-cell responses in rat piriform cortex. Eur J Neurosci 16:2371-82.

Brennan PA, Kendrick KM, Keverne EB. 1995. Neurotransmitter release in the accessory olfactory bulb during and after the formation of an olfactory memory in mice. Neuroscience 69:1075-86.

Brennan PA, Schellinck HM, de la Riva C, Kendrick KM, Keverne EB. 1998. Changes in neurotransmitter release in the main olfactory bulb following an olfactory conditioning procedure in mice. Neuroscience 87:583-90.

Bressler SL. 1988. Changes in electrical activity of rabbit olfactory bulb and cortex to conditioned odor stimulation. Behav Neurosci 102:740-7.

Brunjes PC. 1994. Unilateral naris closure and olfactory system development. Brain Res Brain Res Rev 19:146-60.

Buck LB. 1996. Information coding in the vertebrate olfactory system. Annu Rev Neurosci 19:517-44.
Buonviso N, Gervais R, Chalansonnet M, Chaput M. 1998. Shortlasting exposure to one odour decreases general reactivity in the olfactory bulb of adult rats. Eur J Neurosci 10:2472-5.

Cai Z, Saugstad JA, Sorensen SD, Ciombor KJ, Zhang C, Schaffhauser $\mathrm{H}$, and others. 2001. Cyclic AMP-dependent protein kinase phosphorylates group III metabotropic glutamate receptors and inhibits their function as presynaptic receptors. J Neurochem 78:756-66.

Chaput MA. 2000. EOG responses in anesthetized freely breathing rats. Chem Senses 25:695-701.

Cho JY, Min N, Franzen L, Baker H. 1996. Rapid down-regulation of tyrosine hydroxylase expression in the olfactory bulb of narisoccluded adult rats. J Comp Neurol 369:264-76.

Clark RE, Manns JR, Squire LR. 2002. Classical conditioning, awareness, and brain systems. Trends Cogn Sci 6:524-31.

Connolly JB, Roberts IJ, Armstrong JD, Kaiser K, Forte M, Tully T, O'Kane CJ. 1996. Associative learning disrupted by impaired Gs signaling in Drosophila mushroom bodies. Science 274:2104-7.

Critchley HD, Rolls ET. 1996. Olfactory neuronal responses in the primate orbitofrontal cortex: analysis in an olfactory discrimination task. J Neurophysiol 75:1659-72.

Datiche F, Roullet F, Cattarelli M. 2001. Expression of Fos in the piriform cortex after acquisition of olfactory learning: an immunohistochemical study in the rat. Brain Res Bull 55:95-9.

de Araujo IE, Rolls ET, Kringelbach ML, McGlone F, Phillips N. 2003. Taste-olfactory convergence, and the representation of the pleasantness of flavour, in the human brain. Eur J Neurosci 18:2059-68.

De Rosa E, Hasselmo ME. 2000. Muscarinic cholinergic neuromodulation reduces proactive interference between stored odor memories during associative learning in rats. Behav Neurosci 114:32-41.

Eichenbaum H. 1998. Using olfaction to study memory. Ann N Y Acad Sci 855:657-69.

Engen T. 1991. Odor sensation and memory. New York: Praeger.

Ennis M, Linster C, Aroniadou-Anderjaska V, Ciombor K, Shipley MT. 1998. Glutamate and synaptic plasticity at mammalian primary olfactory synapses. Ann N Y Acad Sci 855:457-66.

Ennis M, Zhou FM, Ciombor KJ, Aroniadou-Anderjaska V, Hayar A, Borrelli E, and others. 2001. Dopamine D2 receptor-mediated presynaptic inhibition of olfactory nerve terminals. J Neurophysiol 86:2986-97.

Fletcher ML, Wilson DA. 2002. Experience modifies olfactory acuity: acetylcholine-dependent learning decreases behavioral generalization between similar odorants. J Neurosci 22:RC201.

Fletcher ML, Wilson DA. 2003. Olfactory bulb mitral-tufted cell plasticity: odorant-specific tuning reflects previous odorant exposure. J Neurosci 23:6946-55.

Frazier-Cierpial L, Brunjes PC. 1989. Early postnatal cellular proliferation and survival in the olfactory bulb and rostral migratory stream of normal and unilaterally odor-deprived rats. J Comp Neurol 289:481-92.

Grajski KA, Freeman WJ. 1989. Spatial EEG correlates of nonassociative and associative olfactory learning in rabbits. Behav Neurosci 103:790-804.

Gray CM, Freeman WJ, Skinner JE. 1986. Chemical dependencies of learning in the rabbit olfactory bulb: acquisition of the transient spatial pattern change depends on norepinephrine. Behav Neurosci 100:585-96.

Haberly LB. 2001. Parallel-distributed processing in olfactory cortex: new insights from morphological and physiological analysis of neuronal circuitry. Chem Senses 26:551-76.

Hasselmo ME, Barkai E. 1995. Cholinergic modulation of activitydependent synaptic plasticity in the piriform cortex and associative memory function in a network biophysical simulation. J Neurosci $15: 6592-604$

Hudson R. 2000. Odor and odorant: a terminological clarification. Chem Senses 25:693.

Hunter AJ, Murray TK. 1989. Cholinergic mechanisms in a simple test of olfactory learning in the rat. Psychopharmacology (Berl) 99:270-5.

Jiang M, Griff ER, Ennis M, Zimmer LA, Shipley MT. 1996. Activation of locus coeruleus enhances the responses of olfactory bulb mitral cells to weak olfactory nerve input. J Neurosci $16: 6319-29$. 
Jung MW, Larson J, Lynch G. 1990. Long-term potentiation of monosynaptic EPSPs in rat piriform cortex in vitro. Synapse 6:279-83.

Kaba H, Nakanishi S. 1995. Synaptic mechanisms of olfactory recognition memory. Rev Neurosci 6:125-41.

Kanter ED, Haberly LB. 1990. NMDA-dependent induction of longterm potentiation in afferent and association fiber systems of piriform cortex in vitro. Brain Res 525:175-9.

Kanter ED, Haberly LB. 1993. Associative long-term potentiation in piriform cortex slices requires GABAA blockade. J Neurosci 13:2477-82.

Kauer JS. 1988. Real-time imaging of evoked activity in local circuits of the salamander olfactory bulb. Nature 331:166-8.

Kay LM, Laurent G. 1999. Odor- and context-dependent modulation of mitral cell activity in behaving rats. Nat Neurosci 2:1003-9.

Leung CH, Wilson DA. 2003. Trans-neuronal regulation of cortical apoptosis in the adult rat olfactory system. Brain Res 984:182-8.

Linster C, Cleland TA. 2002. Cholinergic modulation of sensory representations in the olfactory bulb. Neural Netw 15:709-17.

Linster C, Hasselmo ME. 2000. Neural activity in the horizontal limb of the diagonal band of broca can be modulated by electrical stimulation of the olfactory bulb and cortex in rats. Neurosci Lett 282:157-60.

Linster C, Hasselmo ME. 2001. Neuromodulation and the functional dynamics of piriform cortex. Chem Senses 26:585-94.

Linster C, Johnson BA, Morse A, Yue E, Leon M. 2002. Spontaneous versus reinforced olfactory discriminations. J Neurosci 22:6842-5.

Lipton PA, Alvarez P, Eichenbaum H. 1999. Crossmodal associative memory representations in rodent orbitofrontal cortex. Neuron 22:349-59.

Litaudon P, Mouly AM, Sullivan R, Gervais R, Cattarelli M. 1997. Learning-induced changes in rat piriform cortex activity mapped using multisite recording with voltage sensitive dye. Eur J Neurosci 9:1593-602.

Lledo PM, Gheusi G. 2003. Olfactory processing in a changing brain. Neuroreport 14:1655-63.

MacLeod K, Laurent G. 1996. Distinct mechanisms for synchronization and temporal patterning of odor-encoding neural assemblies. Science 274:976-9.

Martin C, Gervais R, Hugues E, Messaoudi B, Ravel N. 2004 Learning modulation of odor-induced oscillatory responses in the rat olfactory bulb: a correlate of odor recognition? J Neurosci 24:389-97.

Meredith M. 1992. Neural circuit computation: complex patterns in the olfactory bulb. Brain Res Bull 29:111-7.

Mombaerts P. 2001. How smell develops. Nat Neurosci 4 Suppl:1192-8.

Najbauer J, Leon M. 1995. Olfactory experience modulated apoptosis in the developing olfactory bulb. Brain Res 674:245-51.

Nusser Z, Kay LM, Laurent G, Homanics GE, Mody I. 2001. Disruption of GABA(A) receptors on GABAergic interneurons leads to increased oscillatory power in the olfactory bulb network. J Neurophysiol 86:2823-33.

Patneau DK, Stripling JS. 1992. Functional correlates of selective long-term potentiation in the olfactory cortex and olfactory bulb. Brain Res 585:219-28.

Perez-Orive J, Mazor O, Turner GC, Cassenaer S, Wilson RI, Laurent G. 2002. Oscillations and sparsening of odor representations in the mushroom body. Science 297:359-65.

Potter H, Chorover SL. 1976. Response plasticity in hamster olfactory bulb: peripheral and central processes. Brain Res 116:417-29.

Puche AC, Shipley MT. 1999. Odor-induced, activity-dependent transneuronal gene induction in vitro: mediation by NMDA receptors. J Neurosci 19:1359-70.

Ramus SJ, Eichenbaum H. 2000. Neural correlates of olfactory recognition memory in the rat orbitofrontal cortex. J Neurosci 20:8199-208

Ravel N, Chabaud P, Martin C, Gaveau V, Hugues E, Tallon-Baudry C, and others. 2003. Olfactory learning modifies the expression of odour-induced oscillatory responses in the gamma $(60-90 \mathrm{~Hz})$ and beta $(15-40 \mathrm{~Hz})$ bands in the rat olfactory bulb. Eur J Neurosci $17: 350-8$.
Rochefort C, Gheusi G, Vincent JD, Lledo PM. 2002. Enriched odor exposure increases the number of newborn neurons in the adult olfactory bulb and improves odor memory. J Neurosci 22:2679-89.

Rolls ET. 2000. The orbitofrontal cortex and reward. Cereb Cortex 10:284-94.

Roman F, Staubli U, Lynch G. 1987. Evidence for synaptic potentiation in a cortical network during learning. Brain Res 418:221-6.

Roman FS, Chaillan FA, Soumireu-Mourat B. 1993. Long-term potentiation in rat piriform cortex following discrimination learning. Brain Res 601:265-72.

Rosenkranz JA, Grace AA. 2002. Dopamine-mediated modulation of odour-evoked amygdala potentials during pavlovian conditioning. Nature 417:282-7.

Rosselli-Austin L, Altman J. 1979. The postnatal development of the main olfactory bulb of the rat. J Dev Physiol 1:295-313.

Saar D, Barkai E. 2003. Long-term modifications in intrinsic neuronal properties and rule learning in rats. Eur J Neurosci 17:2727-34.

Saar D, Grossman Y, Barkai E. 1999. Reduced synaptic facilitation between pyramidal neurons in the piriform cortex after odor learning. J Neurosci 19:8616-22.

Saar D, Grossman Y, Barkai E. 2001. Long-lasting cholinergic modulation underlies rule learning in rats. J Neurosci 21:1385-92.

Saar D, Grossman Y, Barkai E. 2002. Learning-induced enhancement of postsynaptic potentials in pyramidal neurons. J Neurophysiol $87: 2358-63$.

Savic I. 2002. Brain imaging studies of the functional organization of human olfaction. Neuroscientist 8:204-11.

Schoenbaum G, Eichenbaum H. 1995. Information coding in the rodent prefrontal cortex: I. Single-neuron activity in orbitofrontal cortex compared with that in pyriform cortex. J Neurophysiol 74:733-50.

Scott JW. 1977. A measure of extracellular unit responses to repeated stimulation applied to observations of the time course of olfactory responses. Brain Res 132:247-58.

Serizawa S, Ishii T, Nakatani H, Tsuboi A, Nagawa F, Asano M, and others. 2000. Mutually exclusive expression of odorant receptor transgenes. Nat Neurosci 3:687-93.

Shipley MT, Ennis M. 1996. Functional organization of olfactory system. J Neurobiol 30:123-76.

Slotnick B. 2001. Animal cognition and the rat olfactory system. Trends Cogn Sci 5:216-22.

Slotnick B, Hanford L, Hodos W. 2000. Can rats acquire an olfactory learning set? J Exp Psychol Anim Behav Process 26:399-415.

Spors H, Grinvald A. 2002. Spatio-temporal dynamics of odor representations in the mammalian olfactory bulb. Neuron 34:301-15.

Staubli U, Le TT, Lynch G. 1995. Variants of olfactory memory and their dependencies on the hippocampal formation. J Neurosci 15:1162-71.

Stopfer M, Laurent G. 1999. Short-term memory in olfactory network dynamics. Nature 402:664-8.

Sullivan RM, Stackenwalt G, Nasr F, Lemon C, Wilson DA. 2000. Association of an odor with activation of olfactory bulb noradrenergic beta-receptors or locus coeruleus stimulation is sufficient to produce learned approach responses to that odor in neonatal rats. Behav Neurosci 114:957-62.

Sullivan RM, Wilson DA. 1994. The locus coeruleus, norepinephrine, and memory in newborns. Brain Res Bull 35:467-72.

Sullivan RM, Wilson DA, Leon M. 1989. Norepinephrine and learning-induced plasticity in infant rat olfactory system. J Neurosci 9:3998-4006.

Trombley PQ, Shepherd GM. 1992. Noradrenergic inhibition of synaptic transmission between mitral and granule cells in mammalian olfactory bulb cultures. J Neurosci 12:3985-91.

Wachowiak M, Cohen LB. 1999. Presynaptic inhibition of primary olfactory afferents mediated by different mechanisms in lobster and turtle. J Neurosci 19:8808-17.

Wilson DA. 1998a. Habituation of odor responses in the rat anterior piriform cortex. J Neurophysiol 79:1425-40.

Wilson DA. 1998b. Synaptic correlates of odor habituation in the rat anterior piriform cortex. J Neurophysiol 80:998-1001. 
Wilson DA. 2000. Comparison of odor receptive field plasticity in the rat olfactory bulb and anterior piriform cortex. J Neurophysiol 84:3036-42.

Wilson DA. 2001. Scopolamine enhances generalization between odor representations in rat olfactory cortex. Learn Mem 8:279-85.

Wilson DA. 2003. Rapid, experience-induced enhancement in odorant discrimination by anterior piriform cortex neurons. J Neurophysiol 90:65-72.

Wilson DA, Best AR, Brunjes PC. 2000. Trans-neuronal modification of anterior piriform cortical circuitry in the rat. Brain Res 853:317-22.

Wilson DA, Leon M. 1988. Spatial patterns of olfactory bulb singleunit responses to learned olfactory cues in young rats. J Neurophysiol 59:1770-82.

Wilson DA, Stevenson RJ. 2003. The fundamental role of memory in olfactory perception. Trends Neurosci 26:243-7.

Wilson DA, Sullivan RM. 1995. The D2 antagonist spiperone mimics the effects of olfactory deprivation on mitral/tufted cell odor response patterns. J Neurosci 15:5574-81.

Wilson DA, Sullivan RM, Leon M. 1985. Odor familiarity alters mitral cell response in the olfactory bulb of neonatal rats. Brain Res $354: 314-7$.

Woo CC, Leon M. 1991. Increase in a focal population of juxtaglomerular cells in the olfactory bulb associated with early learning. J Comp Neurol 305:49-56.

Yokoi M, Mori K, Nakanishi S. 1995. Refinement of odor molecule tuning by dendrodendritic synaptic inhibition in the olfactory bulb. Proc Natl Acad Sci U S A 92:3371-5.
Yuan Q, Harley CW, Bruce JC, Darby-King A, McLean JH. 2000. Isoproterenol increases CREB phosphorylation and olfactory nerve-evoked potentials in normal and 5-HT-depleted olfactory bulbs in rat pups only at doses that produce odor preference learning. Learn Mem 7:413-21.

Yuan Q, Harley CW, Darby-King A, Neve RL, McLean JH. 2003. Early odor preference learning in the rat: bidirectional effects of cAMP response element-binding protein (CREB) and mutant CREB support a causal role for phosphorylated CREB. J Neurosci 23:4760-5.

Yuan Q, Harley CW, McLean JH. 2003. Mitral cell beta1 and 5-HT2A receptor colocalization and cAMP coregulation: a new model of norepinephrine-induced learning in the olfactory bulb. Learn Mem 10:5-15.

Zinyuk LE, Datiche F, Cattarelli M. 2001. Cell activity in the anterior piriform cortex during an olfactory learning in the rat. Behav Brain Res 124:29-32.

Zou DJ, Greer CA, Firestein S. 2002. Expression pattern of alpha CaMKII in the mouse main olfactory bulb. J Comp Neurol 443:226-36.

Zou Z, Horowitz LF, Montmayeur JP, Snapper S, Buck LB. 2001. Genetic tracing reveals a stereotyped sensory map in the olfactory cortex. Nature 414:173-9.

Zufall F, Leinders-Zufall T. 2000. The cellular and molecular basis of odor adaptation. Chem Senses 25:473-81.

Zufall F, Shepherd GM, Firestein S. 1991. Inhibition of the olfactory cyclic nucleotide gated ion channel by intracellular calcium. Proc R Soc Lond B Biol Sci 246:225-30. 